
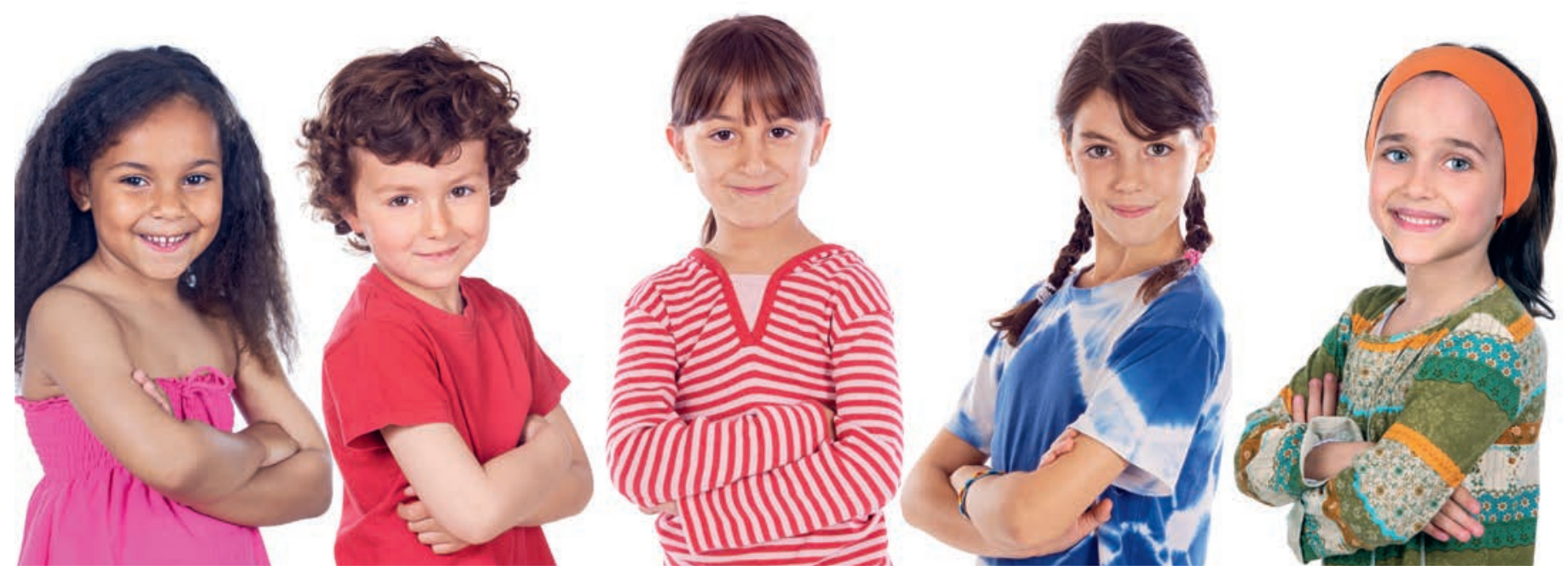

Plädoyer für eine qualitativ hochstehende Kinder- und Jugendmedizin in der Schweiz

\title{
Kinder sind keine kleinen Erwachsenen!
}

\section{Gian Paolo Ramellia, Alain di Gallob, Stefan Holland-Cunz ${ }^{\mathrm{c}}$}

a Prof. Dr. med., Präsident Schweizerische Gesellschaft für Pädiatrie SGP; b Prof. Dr. med., Co-Präsident Schweizerische Gesellschaft für Kinder- und Jugendpsychiatrie und -psychotherapie SGKJPP/SSPPEA; ${ }^{\circ}$ Prof. Dr. med., Präsident Schweizerische Gesellschaft für Kinderchirurgie SGKC

\section{Hinweis}

Der Text wurde im Namen der Expertengruppe Kinderund Jugendmedizin verfasst, welche das Positions papier erarbeitet hat.

Diesem Satz würde jeder und jede beipflichten. Im Gesundheitswesen sieht es anders aus: Kinder und ihre Angehörigen bedürfen oft mehr Zeit und eines anderen Zugangs als in unseren Systemen vorgesehen. Nationale Parlamentarierinnen und Parlamentarier haben die Problematik erkannt und setzen sich für politische Lösungen ein.

Sowohl in der somatischen als auch in der psychiatrischen Kinder- und Jugendmedizin schreiben Spitäler und Ambulatorien Defizite, und in den Praxen besteht, besonders abseits der städtischen Zentren, ein akutes Nachwuchsproblem. Wir müssen uns diesen Herausforderungen stellen, sonst ist die medizinische Versorgung der jüngsten Patientinnen und Patienten gefährdet, die wesentliche gesundheitliche, soziale und ökonomische Weichen für den weiteren Lebensweg stellt.

Nationale Parlamentarierinnen und Parlamentarier haben die Problematik erkannt und am 24. September 2018 unter dem Co-Präsidium der Nationalrätinnen Marina Carobbio Guscetti, Verena Herzog, Ruth Humbel, Tiana Moser und Ständerat Damian Müller die parlamentarische Gruppe Kinder- und Jugendmedizin gegründet. Die Gruppe hat sich zum Ziel gesetzt, politische Lösungen für eine qualitativ hochstehende zukünftige Kinder- und Jugendmedizin in der
Schweiz zu erarbeiten. Das Co-Präsidium erteilte einer Expertengruppe den Auftrag, Kernargumente zu konkretisieren, Handlungsfelder zu beschreiben und politische Forderungen abzuleiten. Die aus Fachpersonen der Pädiatrie, Kinderchirurgie, Kinder- und Jugendpsychiatrie und -psychologie, pädiatrischen Pflege und Gesundheitspolitik zusammengesetzte Gruppe erarbeitete in der Folge ein gemeinsames Positionspapier, das am 9. September 2019 veröffentlicht wurde [1]. Es umfasst sieben Leitpunkte, die mit je einem Beispiel und einer konkreten Forderung dargestellt werden.

\section{Die Behandlung von Kindern und Jugend- lichen benötigt genug Zeit}

Minderjährige sind besonders schutzbedürftig. Sie müssen altersgerecht aufgeklärt und angemessen in die medizinische Behandlung einbezogen werden. Ohne 
Verständnis für die spezifischen Bedürfnisse des Kindes und ohne Kenntnis seines Entwicklungsstandes sind Kommunikation und Handeln nicht möglich. Beispiel: Ein Vater sucht mit seinem dreijährigen Sohn wegen eines Asthmaanfalls die Praxis der Kinderärztin auf. Nur dank viel Geduld und Zeit der Ärztin und ihrer Assistentin verliert der Knabe die Angst vor dem Inhalieren. Die Geduld lohnt sich. Sie verhindert eine Hospitalisation und spart viel Stress für Kind und Eltern und auch viel Geld.

Forderung: Das Tarifsystem muss den Zeitbedarf für die Behandlung von Kindern adäquat berücksichtigen.

\section{Der Einsatz für die Gesundheit der Kinder lohnt sich langfristig}

Eine ausreichende Grundversorgung in allen Regionen ist unverzichtbar und hilft Folgekosten zu vermeiden. In der Pädiatrie und der Kinder- und Jugendpsychiatrie besteht bereits heute in einigen Regionen eine akute Unterversorgung. Der altersbedingte Rückzug vieler Praxisinhaberinnen und -inhaber und die Zunahme der Teilzeitarbeit werden die Notlage in den kommenden Jahren verschärfen.

Beispiel: Ein Kinderarzt in einer ländlichen Gemeinde findet keine Nachfolgerinnen und Nachfolger. Um sein bisheriges Arbeitspensum abzudecken und seine Patientinnen und Patienten angemessen zu versorgen, sind zwei bis drei Personen notwendig.

Forderung: Der Bund muss eine Versorgungsforschung für alle kindermedizinischen Disziplinen initiieren, damit eine Planung möglich und die Schweiz für die Zukunft gerüstet ist.

\section{Der Einbezug des Lebensumfeldes ist entscheidend}

Kinder und Jugendliche sind immer Teil eines sozialen Systems. Um sie adäquat behandeln zu können, ist der Einbezug der Eltern resp. der sorgeberechtigten Erwachsenen, der Lehrerinnen und Lehrer sowie, je nach Situation, zusätzlicher Bezugspersonen unerlässlich. Beispiel: Ein sechsjähriges Mädchen nässt plötzlich wieder ein. Im Gespräch mit den Eltern erfährt der Kinderpsychiater, dass eine Trennung der Eltern im Raum steht, die Tochter aber noch nichts davon wissen soll. Das Kind könnte mit seinem Symptom einen unausgesprochenen Konflikt nach aussen tragen. Ohne intensiven Einbezug der Eltern ist eine Behandlung sinnlos.

Forderung: Der Aufwand für den Einbezug des Umfeldes, gegebenenfalls inklusive Dolmetscher, muss ohne Limitationen vergütet werden.

\section{Hohe Ansprüche an die Ausgestaltung der Infrastruktur}

Die Kindermedizin behandelt kleinste Patienten mit kaum 500 g Körpergewicht und bis zu zwei Meter grosse Jugendliche mit weit über 100 kg Körpergewicht. Das entwicklungspsychologische Spektrum reicht vom völlig abhängigen Säugling bis zum Jugendlichen in der Berufsfindung. Um dieser Vielfalt gerecht zu werden, ist die Bereitstellung einer altersgemässen Umgebung und grössenentsprechender Materialien notwendig.

Beispiel: Ein Kinderspital führt aus Kostengründen nur zwei Urinkathetergrössen: eine für Säuglinge und eine für Erwachsene. Bei einem fünfährigen Knaben mit Harnverhalt muss anstelle eines Urinkatheters eine Magensonde verwendet werden, weil der Säuglingskatheter rausrutscht und der Erwachsenenkatheter viel zu gross ist. Die Magensonde kann für diese fachfremde Anwendung der Versicherung nicht in Rechnung gestellt werden.

Forderung: Die notwendige kindergerechte Infrastruktur ist bereitzustellen und zu vergüten. Auch zweckmässige Umnutzungen von Materialien müssen vergütet werden.

\section{Koordination zwischen den beteiligten Kostenträgern bei Therapien ungenügend}

Die Kostenzuständigkeit für die Behandlung vieler Krankheiten im Kindes- und Jugendalter liegt bei verschiedenen Trägern. Daraus resultierende Fragen zu Indikationen und Kostengutsprachen werden auf Eltern, Ärztinnen und Ärzte sowie Spitäler übertragen. Im schlimmsten Fall führt dies zu Behandlungsverzögerungen oder gar Behandlungsablehnungen.

Beispiel: Ein dreijähriges autistisches Mädchen aus dem Kanton Aargau könnte für seine spätere soziale und schulische Integration sehr von einer intensiven Frühintervention profitieren. Für diese spezialisierte Behandlung gibt es in der Schweiz fünf Zentren. Gemäss eidgenössischem Finanzausgleich bezahlt die IV die medizinischen Leistungen, die Kantone die heilpädagogischen Leistungen dieses interprofessionellen Settings. Viele Kantone lehnen es ab, ausserkantonale Kosten zu übernehmen, auch wenn es im eigenen Kanton kein entsprechendes Angebot gibt. Das Mädchen erhält keine Therapie, obwohl es in Basel ein Angebot gäbe, das ihm helfen könnte.

Forderung: Bund, Kantone und Versicherungen richten eine Koordinationskommission ein, die strittige $\mathrm{Zu}$ ständigkeiten klärt. Besteht eine dringende medizinische Indikation, darf die Therapie vor der endgültigen Einigung durchgeführt werden. 


\section{Kinderspezifische Heilmittel haben wenig Marktpotential}

Das Problem der fehlenden Arzneimittel und Impfstoffe für Kinder und Jugendliche nimmt zu. Einerseits werden immer mehr kindgerechte Applikationsformen vom Markt genommen, andererseits erschweren hohe nationale Hürden die Einführung von im Ausland zugelassenen Medikamenten.

Beispiel: Drei Kinder einer Familie leiden an juckender Krätze. Nach verschiedenen vergeblichen Therapieversuchen wird eine Behandlung mit einem speziellen Antibiotikum erfolgreich eingesetzt. Dieses Medikament ist in den umliegenden Ländern, nicht aber in der Schweiz zugelassen. Die Krankenkasse lehnt die Kostenübernahme ab.

Forderung: Der Bundesrat und die Gesundheitsbehörden setzen sich dafür ein, dass auch im Bereich der Kinderarzneimittel Zulassungen gefördert werden. Der Off-Label- und der Unlicensed-Use, die in der Kinder- und Jugendmedizin Alltag sind, müssen klar geregelt werden

\section{Die Arbeit in der Kinder- und Jugend- medizin muss nachhaltig attraktiv sein}

Immer weniger Medizinstudentinnen und -studenten entscheiden sich für eine Weiterbildung in Kinderund Jugendpsychiatrie. In der pädiatrischen Pflege besteht ein grosser Mangel an Fachpersonal, und es gibt keine spezifische Ausbildung mehr.

Beispiel: Eine junge Frau absolviert eine Lehre als Fachangestellte Gesundheit. Sie möchte später mit Kindern arbeiten, findet jedoch während ihrer Lehre keinen Praktikumsplatz in einem Kinderspital. Auch im Theorieunterricht erhält sie kaum kinderspezifisches Wissen vermittelt. Nach Abschluss der Lehre bekommt sie eine Anstellung in einem Kinderspital. Sie nimmt sie mit Freude an, fühlt sich aber überfordert, weil sie zu wenig weiss, und gibt den Beruf frustriert wieder auf. Forderung: Bei der Zulassungsprüfung zum Medizinstudium müssen Fähigkeiten, die für die Kinder- und Jugendmedizin und -psychiatrie wichtig sind, stärker berücksichtigt werden. In der Pflegeausbildung ist eine Spezialisierung für die Kinder- und Jugendmedizin von Beginn weg aufzubauen.

Diese für die Kinder- und Jugendmedizin wegweisenden Fakten müssen dringend in die zukünftigen gesundheitspolitischen Diskussionen und Entscheidungen einbezogen werden. Einige der genannten Punkte sind in beschränktem Mass auch für die Erwachsenenmedizin gültig. Es geht nicht darum, die Kindermedizin gegen die übrigen Disziplinen auszuspielen. Ziel des Positionspapiers ist es, auf Mängel hinzuweisen, die in der Vergangenheit nicht ausreichend erkannt und, weil die finanziellen Defizite der Kindermedizin oft in der Rechnung von Klinikverbünden verborgen blieben, zu wenig beachtet wurden.

Mitglieder des Stände- und des Nationalrats haben in der Herbstsession mehrere parlamentarische Vorstösse eingereicht, um die genannten Probleme anzupacken. So wird der Bundesrat u.a. beauftragt, in $\mathrm{Zu}$ sammenarbeit mit den Kantonen, den betroffenen Organisationen und Fachpersonen eine nationale Strategie zur Stärkung der Kinder- und Jugendmedizin zu erarbeiten. Die Expertengruppe Kinder- und Jugendmedizin ist gerne bereit, die Arbeiten zu begleiten und ihre Expertise einzubringen.

\section{Bildnachweis}

๔ J Jose Manuel Gelpi Diaz | Dreamstime.com (Symbolbild)

\section{Literatur}

1 https://paediatrica.swiss-paediatrics.org/parlamentarischegruppe/

- Die parlamentarische Gruppe Kinder- und Jugendmedizin wurde im September 2018 gegründet.

- Sie hat sich zum Ziel gesetzt, politische Lösungen für eine qualitativ hochstehende zukünftige Kinder- und Jugendmedizin in der Schweiz zu erarbeiten.

- Das Co-Präsidium erteilte einer Expertengruppe den Auftrag, Kernargumente zu konkretisieren, Handlungsfelder zu beschreiben und politische Forderungen abzuleiten.

- Im vergangenen Jahr hat die Expertengruppe ein gemeinsames Positionspapier erarbeitet.

- Das Positionspapier wurde am 9. September 2019 veröffentlicht; es umfasst sieben Leitpunkte, dargestellt mit je einem konkreten Beispiel und einer konkreten Forderung.

\section{L'essentiel en bref}

- L'intergroupe parlementaire Médecine pédiatrique a été créé en septembre 2018

- Il a pour but d'élaborer des solutions politiques pour une future médecine pédiatrique de haute qualité en Suisse.

- La coprésidence a chargé un groupe d'experts de concrétiser les arguments clés, de décrire les domaines d'action et d'en dériver des revendications politiques.

- L'an dernier, ce groupe d'experts a rédigé une prise de position commune.

- Publiée le 9 septembre 2019, elle comporte sept points clés, chacun accompagné d'un exemple et d'une revendication concrets. 